\title{
A Tool for Intersecting Context-Free Grammars and Its Applications
}

\author{
Graeme Gange $^{1}$, Jorge A. Navas ${ }^{2}$, Peter Schachte ${ }^{1}$, Harald Søndergaard ${ }^{1}$, and \\ Peter J. Stuckey ${ }^{1}$ \\ 1 The University of Melbourne, Vic. 3010, Australia \\ 2 NASA Ames Research Center, Moffett Field, CA 94035, USA
}

\begin{abstract}
This paper describes a tool for intersecting context-free grammars. Since this problem is undecidable the tool follows a refinementbased approach and implements a novel refinement which is complete for regularly separable grammars. We show its effectiveness for safety verification of recursive multi-threaded programs.
\end{abstract}

\section{Introduction}

Checking emptiness of intersections of context-free grammars is a well-known undecidable problem. However, the fact that this problem is equivalent to safety verification of recursive multi-threaded programs has kept motivating the design of semi-decision procedures that can still be effective in practice.

In this paper, we describe COVENANT, a tool for checking whether the languages of an arbitrary number of context-free grammars are disjoint and show its role as a component in the analysis of recursive multi-threaded programs. The tool takes a grammatical approach [7], in the sense that it is formalized in terms of context-free grammars rather than pushdown automata $[1,2,10]$. It implements a counter-example guided abstraction refinement (CEGAR) of regular over-approximations and integrates a complete refinement procedure that guarantees termination if the context-free grammars are regularly separable. ${ }^{3} \mathrm{We}$ show its application to safety verification of recursive multi-threaded programs.

To the best of our knowledge, our tool is the only publicly available implementation tackling the problem of intersecting unbounded context-free grammars.

\section{Approach}

The tool discussed in this paper follows the so-called counter-example guided abstraction refinement (CEGAR) of regular over-approximations. Without loss of generality, in this presentation we consider the intersection of just two contextfree grammars $G_{1}$ and $G_{2}$. The scheme is based on an initial abstraction which is repeatedly refined until either the languages are proven disjoint, an intersection witness has been found, or resources have been exhausted:

\footnotetext{
${ }^{3}$ Two context-free grammars $G_{1}$ and $G_{2}$ are regularly separable if there exist two regular languages $L_{1}$ and $L_{2}$ such that $\mathcal{L}\left(G_{1}\right) \subseteq L_{1}, \mathcal{L}\left(G_{2}\right) \subseteq L_{2}$ and $L_{1} \cap L_{2}=\emptyset$.
} 
1. Abstraction: compute regular approximations $R_{1}$ and $R_{2}$ such that $\mathcal{L}\left(G_{1}\right) \subseteq$ $\mathcal{L}\left(R_{1}\right)$ and $\mathcal{L}\left(G_{2}\right) \subseteq \mathcal{L}\left(R_{2}\right)$.

2. Verification: using a decision procedure for regular languages if $\mathcal{L}\left(R_{1}\right) \cap$ $\mathcal{L}\left(R_{2}\right)=\emptyset$ then $\mathcal{L}\left(G_{1}\right) \cap \mathcal{L}\left(G_{2}\right)=\emptyset$, so answer "the languages are disjoint." If $w \in\left(\mathcal{L}\left(R_{1}\right) \cap \mathcal{L}\left(R_{2}\right)\right), w \in \mathcal{L}\left(G_{1}\right)$, and $w \in \mathcal{L}\left(G_{2}\right)$ then $\mathcal{L}\left(G_{1}\right) \cap \mathcal{L}\left(G_{2}\right) \neq$ $\emptyset$, so answer "the languages are not disjoint" and provide $w$ as a witness. Otherwise, go to step 3.

3. Refinement: produce new regular approximations $R_{1}^{\prime}$ and $R_{2}^{\prime}$ such that for each $R_{i}^{\prime}, i \in\{1,2\}$, we have $\mathcal{L}\left(G_{i}\right) \subseteq \mathcal{L}\left(R_{i}^{\prime}\right) \subseteq \mathcal{L}\left(R_{i}\right)$, and $\mathcal{L}\left(R_{i}^{\prime}\right) \subset \mathcal{L}\left(R_{i}\right)$ for some $i$. Update the approximations $R_{1} \leftarrow R_{1}^{\prime}, R_{2} \leftarrow R_{2}^{\prime}$, and go to step 2 .

Abstraction. Note that a regular approximation always exists for any grammar $G$ since we can use $\Sigma^{*}$, where $\Sigma$ is the alphabet of $G$. However, the precision of the initial abstraction often has a significant impact on the convergence of the refinement loop, so non-trivial initial abstractions such as the $i^{t h}$-prefix abstraction $[1,2]$ and the downward closure with a cycle-breaking heuristic [7] are more suitable candidates.

Verification. This step assumes a decision procedure that returns "no" if $\mathcal{L}\left(A_{1}\right) \cap$ $\mathcal{L}\left(A_{2}\right)=\emptyset$ or returns a witness $w$ if $w \in \mathcal{L}\left(A_{1}\right) \cap \mathcal{L}\left(A_{2}\right) \neq \emptyset$, where $A_{1}$ and $A_{2}$ are finite-state automata recognizing regular languages $R_{1}$ and $R_{2}$, respectively (that is, $\mathcal{L}\left(A_{1}\right)=R_{1}$ and $\mathcal{L}\left(A_{2}\right)=R_{2}$ ). This can be solved using, for instance, the classical product construction. Note that a different approach would make use of the fact that the class of context-free languages is closed under intersection with regular languages. However, one advantage of our approach is that we are able to leverage the latest advances made in string solving $[4,6,11]$.

Refinement. At this point, the regular solver has found some witness $w$ such that $w \in\left(\mathcal{L}\left(A_{1}\right) \cap \mathcal{L}\left(A_{2}\right)\right)$, but $w \notin\left(\mathcal{L}\left(G_{1}\right) \cap \mathcal{L}\left(G_{2}\right)\right)$. There are three cases: (1) $w \notin \mathcal{L}\left(G_{1}\right) \wedge w \in \mathcal{L}\left(G_{2}\right),(2) w \notin \mathcal{L}\left(G_{1}\right) \wedge w \notin \mathcal{L}\left(G_{2}\right)$, and $(3) w \in \mathcal{L}\left(G_{1}\right) \wedge w \notin$ $\mathcal{L}\left(G_{2}\right)$. For (1) and (3) we should refine $A_{1}$ and $A_{2}$, respectively. For (2) we could choose to refine either $A_{1}$ or $A_{2}$, or both. COVENANT aggressively refines both.

We say a language $L$ is a safe generalization of a witness $w$ with respect to a context-free grammar $G$ if (a) $L \supseteq\{w\}$ and (b) $L \cap \mathcal{L}(G)=\emptyset$. If $w \notin$ $\mathcal{L}\left(G_{i}\right)$ then a straightforward refinement is to produce a new abstraction that recognizes $\mathcal{L}\left(A_{i}\right) \backslash\{w\}$ in place of $A_{i}$. However, that refinement process will rarely converge, as it excludes only finitely many examples. Instead, we would like to produce safe generalizations of $w$ containing an infinite number of words, to hasten convergence. For this purpose, our tool implements the concept of stargeneralization [5]. Informally, a star-generalization of a word $w$ is a language that applies the Kleene $*$ operator (i.e., unbounded repetition) to any number of non-overlapping, but possibly nested, subsequences of $w$, while ensuring the resulting augmented language remains disjoint with the language of $G$.

\section{Covenant}

The tool is publicly available at https ://bitbucket.org/jorgenavas/covenant. 


\subsection{Design and Implementation Choices}

The tool is implemented in $\mathrm{C}++$ and parameterized by the initial approximation, the regular solver, and the refinement procedure.

Abstraction. One advantage of having a grammatical view is that COVENANT can easily leverage the advances made in areas such as speech processing where precise abstraction of context-free grammars into regular grammars is an active topic of research. COVENANT implements the method described by Nederhof [8] for approximating context-free grammars with strongly regular languages.

We say a grammar is strongly regular if all productions are of the form: $A \rightarrow B w \mid w$ or $A \rightarrow w B \mid w$, where $w \in \Sigma^{*}$ and $A, B$ are nonterminals. The abstraction relies on the following observation: a grammar with productions of the form $A \rightarrow \alpha A \beta$ with both $\alpha, \beta$ non-empty might not be represented as a strongly regular grammar because $\alpha$ and $\beta$ might be related through an "unbounded" communication not expressible by regular languages. The abstraction consists of breaking conservatively those unbounded communications in such a way that the grammar becomes regular while preserving as much as possible the structure of the original grammar. Nederhof [8] also proposed a transformation from strongly regular grammars to finite automata also implemented in COVENANT.

Regular Solver. COVENANT currently implements only the naive product construction for intersecting finite automata but other regular solvers can easily be plugged in. In fact, an initial implementation of COVENANT was tested using REVENANT [4], an efficient regular solver based on bounded model checking with interpolation, though the released version does not incorporate it.

Refinement. COVENANT implements both the greedy and maximum star-epsilon generalizations both described in [5]. We refer to [5] for details and describe them informally through an example. Assume we have a witness $w \equiv$ aab and a context-free language $L=\left\{\mathrm{a}^{i} \mathrm{~b}^{i+1} \mid i \geq 0\right\}$. The greedy algorithm starts by checking whether $W_{1} \equiv \mathrm{a}^{*} \mathrm{ab} \notin L$. Since the query succeeds, $W_{1}$ is already a safe generalization of $w$ wrt. $L$ so we could stop here. However, we can continue and check next whether $W_{2} \equiv \mathrm{a}^{*} \mathrm{a}^{*} \mathrm{~b} \notin L$ but $\mathrm{b} \in L$ so $W_{2}$ must be discarded. Next, we try whether $W_{3} \equiv \mathrm{a}^{*} \mathrm{ab}^{*} \notin L$ but abb $\in L$ and thus $W_{3}$ is also not a safe generalization. Finally, we query $W_{4} \equiv \mathrm{a}^{*}(\mathrm{ab})^{*} \notin L$ and $W_{5} \equiv\left(\mathrm{a}^{*}(\mathrm{ab})^{*}\right)^{*} \notin L$ which both succeed. Therefore, starting from aab, our tool can produce the safe generalization $\left(\mathrm{a}^{*}(\mathrm{ab})^{*}\right)^{*}$. In fact, our tool generated three safe generalizations: $W_{1} \subseteq W_{4} \subseteq W_{5}$. Although this greedy method is reasonably cheap $\left(O\left(|w|^{2}\right)\right)$, if resources are scarce it can stop at any time, returning either $W_{1}$ or $W_{4}$.

The maximum star-epsilon version is similar to the greedy one except it will compute the union of all possible safe generalizations without committing to any successful partial generalization. That is, the greedy version started by checking $W_{1}$ and since $W_{1}$ succeeded the rest of queries were relative to $W_{1}$. The non-greedy version will not commit to $W_{1}$ but also try other possibilities. For instance, $a a^{*} b,(a a)^{*} b,(a a b)^{*}$, and $a(a b)^{*}$ are also safe generalizations from which we can keep generalizing. Although more expensive, it is worth mentioning 
that this version ensures termination of the CEGAR loop whenever the contextfree grammars are regularly separable.

For the implementation, two operations are important: (a) intersection between a context-free grammar and a finite automaton for checking safe generalizations, and (b) automata difference for refining the current abstraction by discarding a safe generalization $W$ of $w$. For (a), COVENANT uses a modified version of the efficient pre* algorithm [3] and for (b) it intersects the current abstraction with the complement of the determinization of $W$. Although determinization of automata can have an exponential size blowup, this behavior is rare; we have not seen it during experiments. Based on our experience, it is also useful to minimize after (b) has been performed, to keep the abstraction small.

\subsection{Preprocessing and Output}

We require that the input grammars are in the following normal form: $A \rightarrow B C$ | $B|a| \varepsilon$, where $A, B, C$ are nonterminals and $a \in \Sigma^{+}$, where $\Sigma$ is the alphabet of the grammar. Any context-free grammar can be converted to this form by a linear increase in terms of the size of the original grammar. COVENANT performs this normalization as a preprocessing step but it does not require any further (more expensive) normalizations, such as Chomsky Normal Form.

If COVENANT proves that the language of the grammars are not disjoint it will return a witness. The user can set the option --solutions $\mathrm{n}$ to ask the solver for $n$ solutions. The option --dot will output the automata resulting from the initial abstraction, each of the safe generalizations, and the final abstractions when emptiness was proven, all in the dot language of the Graphviz package.

\section{Safety Verification of Multithread Programs}

Bouajjani et al. [1] pioneered safety verification of recursive multi-threaded programs by reduction to checking the intersection of context free languages for emptiness. For lack of space, we refer to $[1,2,7]$ for details of the encoding.

We have tested COVENANT and compared with LCEGAR [7] using two classes of programs: textbook Erlang programs and several variants of a real Bluetooth driver. A detailed description of the programs as well as the safety properties can be found in $[2,7,9]$. We ran LCEGAR with the setting provided by the authors and tried with the two available initial abstractions: pseudo-downward closure $(\mathrm{PDC})$ and cycle breaking $(\mathrm{CB})$. Table 1 shows the results. The symbol $\infty$ means a timeout expired after 2 hours. We ran COVENANT with the greedy refinement.

\section{Related Work}

To the best of our knowledge, COVENANT is the first publicly available implementation for intersecting context-free grammars ensuring termination for regularly separable grammars. Several CEGAR approaches have been proposed before. 


\begin{tabular}{|l|c||c||c|c|}
\hline \multicolumn{2}{|c||}{ Program } & COVENANT & \multicolumn{2}{c|}{ LCEGAR } \\
\hline \multicolumn{2}{|c||}{} & & PDC & CB \\
\hline \hline SharedMem & safe & 0.01 & 14.37 & 24.75 \\
\hline Mutex & safe & 0.04 & 6.12 & 0.14 \\
\hline RA & safe & 0.01 & $\infty$ & 0.39 \\
\hline Modified RA & safe & 0.03 & $\infty$ & 27.90 \\
\hline TNA & unsafe & 0.01 & 0.02 & 0.25 \\
\hline Banking & unsafe & 0.01 & $\infty$ & 3.36 \\
\hline
\end{tabular}

(a) Verification of multi-thread Erlang programs

\begin{tabular}{|l|c||r||r|r|}
\hline \multicolumn{2}{|c||}{ Program } & \multicolumn{1}{c||}{ COVENANT } & \multicolumn{2}{c|}{ LCEGAR } \\
\hline \multicolumn{2}{|l||}{} & & PDC & CB \\
\hline \hline Version 1 & unsafe & 0.84 & 19.74 & 21.04 \\
\hline Version 2 & unsafe & 0.25 & 5560.00 & 4852.00 \\
\hline Version 2 w/ Heuri & unsafe & 0.11 & 44.68 & 38.89 \\
\hline Version 3 (1A2S) & unsafe & 0.12 & 217.74 & 217.27 \\
\hline Version 3 (1A2S) w/ Heuri & unsafe & 0.05 & 6.68 & 11.37 \\
\hline Version 3 (2A1S) & safe & 0.27 & 4185.00 & 3981.00 \\
\hline
\end{tabular}

(b) Verification of multi-thread Bluetooth drivers

Table 1: Comparison of COVEnAnT with LCEGAR; times in seconds. All experiments ran on a single core of a $2.4 \mathrm{GHz}$ Core i5-M520 with $8 \mathrm{GiB}$ of memory.

Here, we do not consider the effect of initial approximations, as they do not affect the expressiveness of the refinement loop and are easily interchangeable.

The first CEGAR approach was proposed in [1] based on the concept of refinable finite-chain abstraction which consists of computing the series $\left(\alpha_{i}\right)_{i \geq 1}$ overapproximating the language of a CFG $G$ such that $\mathcal{L}\left(\alpha_{1}(G)\right) \supset \mathcal{L}\left(\alpha_{2}(G)\right) \supset$ $\ldots \supseteq \mathcal{L}(G)$. Several refinable abstractions were described in [1] although no experimental data was provided. Instead, we compare here with the $i^{\text {th }}$-prefix abstraction $^{4}$ implemented in [2]. In this, $\alpha_{i}(G)$ is the set of words of $G$ of length less than $i$, together with the set of prefixes of length $i$ of $G$. We argue that the refinement implemented in COVENANT is more expressive as it is not hard to find regularly separable languages that cannot be proven so by the $i^{\text {th }}$-prefix abstraction. For instance, with $R_{1} \equiv \mathrm{a}^{*} \mathrm{~b}$ and $R_{2} \equiv \mathrm{a}^{*} \mathrm{c}$, we have $R_{1} \cap R_{2}=\emptyset$, while for every length $i$, the string $a^{i}$ forms a prefix to words in both $R_{1}$ and $R_{2}$. Therefore the intersection of the two abstractions will always be non-empty.

The LCEGAR method described in [7] is based on a similar refinement framework, but the approach differs radically. LCEGAR maintains a pair of context-free grammars $A_{1}, A_{2}$, over-approximating the intersection of the original languages. At each refinement step, an elementary bounded language $B_{i}$ is generated from each grammar $A_{i} .{ }^{5}$ The refinement ensures $B_{i} \cap A_{i} \neq \emptyset$, but $B_{i}$ is not necessarily

4 [2] also implemented the $i^{\text {th }}$-suffix abstraction which suffers from same limitations.

${ }^{5}$ An elementary bounded language is a language of the form $B=w_{1}^{*} \ldots w_{k}^{*}$, where each $w_{i}$ is a (finite) word in $\Sigma^{*}$. 
either an over- or under-approximation of $A_{i}$. After that, $I=B_{i} \cap L_{1} \cap L_{2}$ is computed. If $I$ is non-empty, $L_{1} \cap L_{2}$ must also be non-empty. If $I$ is empty, then the approximations can safely be refined by removing $B_{i}$.

Here a comparison between methods is more involved, and we refer to [5] for details. Suffice it to say that the refinements done by LCEGAR and COVENANT are incomparable. That is, there are grammars which are not regularly separable for which LCEGAR can terminate but COVENANT cannot, and there are also grammars which are regularly separable but LCEGAR cannot terminate.

Finally, the verification phase in COVENANT consists of intersecting finite automata, for which efficient solvers are available. Instead, LCEGAR intersects several context-free grammars and a bounded language which, although decidable, is NP-Complete. In our experience, LCEGAR makes a smaller number of refinements than COVENANT, but each refinement in COVENANT is considerably cheaper than in LCEGAR, resulting in the better performance.

\section{Conclusions}

The main contributions of this paper have been to describe a tool for intersecting context-free grammars, and to show it can be effective for safety verification of recursive multi-threaded programs.

\section{References}

1. A. Bouajjani, J. Esparza, and T. Touili. A generic approach to the static analysis of concurrent programs with procedures. In POPL, pages 62-73, 2003.

2. S. Chaki, E. Clarke, N. Kidd, T. Reps, and T. Touili. Verifying concurrent messagepassing C programs with recursive calls. In TACAS, pages 334-349, 2006.

3. J. Esparza, P. Rossmanith, and S. Schwoon. A uniform framework for problems on context-free grammars. Bulletin of the EATCS, 72:169-177, 2000.

4. G. Gange, J. A. Navas, P. J. Stuckey, H. Søndergaard, and P. Schachte. Unbounded model-checking with interpolation for regular language constraints. In TACAS, pages 277-291, 2013.

5. G. Gange, J. A. Navas, P. J. Stuckey, H. Søndergaard, and P. Schachte. A complete refinement procedure for regular separability of context-free languages. Technical report, The University of Melbourne, http://people.eng.unimelb.edu.au/ gkgange/pubs/cfg_preprint.pdf, November 2014.

6. P. Hooimeijer and W. Weimer. StrSolve: Solving string constraints lazily. ASE, 19(4):531-559, 2012.

7. Z. Long, G. Calin, R. Majumdar, and R. Meyer. Language-theoretic abstraction refinement. In FASE, pages 362-376, 2012.

8. M.-J. Nederhof. Regular approximation of CFLs: A grammatical view. In Advances in Probabilistic and Other Parsing Technologies, volume 16, pages 221-241. 2000.

9. S. Qadeer and D. Wu. KISS: Keep it simple and sequential. In PLDI, pages 14-24, 2004.

10. D. Suwimonteerabuth, S. Schwoon, and J. Esparza. jMoped: A Java bytecode checker based on Moped. In TACAS, pages 541-545, 2005.

11. M. Veanes, P. de Halleux, and N. Tillmann. Rex: Symbolic regular expression explorer. In $I C S T V V$, pages 498-507, 2010. 


\section{Appendix: Tool Demonstration}

Installation. The installation process relies on cmake which allows COVENANT being installed across different platforms ${ }^{6}$. The only requirements are:

- to install the boost library

- to install a reasonable modern $\mathrm{C}++$ compiler version. COVENANT has been tested for clang++ 3.2 and $\mathrm{g}++4.8$.

Usage. The options for running COVENANT are:

covenant [OPTIONS] INPUT

where INPUT is a text file containing the context-free grammars to be intersected. We will describe the most relevant options as they are needed during this presentation. All options are visible by typing covenant $-\mathrm{h}$.

"UNSAT" example. Consider the two contrived regularly-separable context-free languages $L_{1}=\left\{w \mathrm{c} w^{R} \mid w \in\{\mathrm{a}, \mathrm{b}\}^{*}\right\}$ and $L_{2}=\left\{\mathrm{a}^{n} \mathrm{cb}^{n}\right\}$. The content of INPUT is the following:

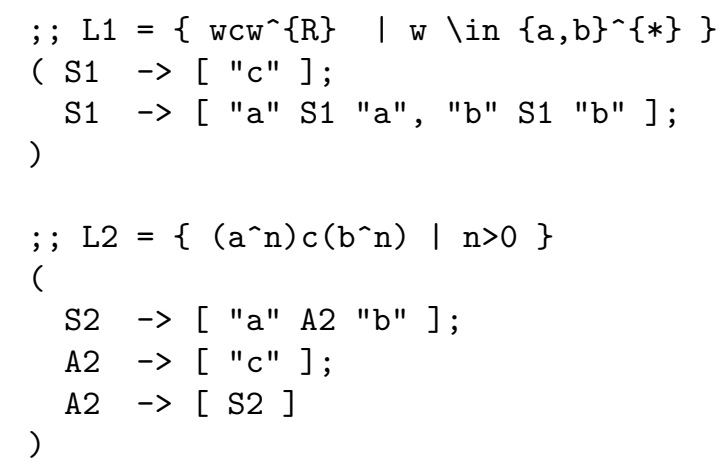

The nonterminal symbol appearing on the left-hand side in the first grammar production is considered the start symbol of the grammar. In the above example the start symbols are S1 and S2, respectively. Terminal symbols must be between double quotes (e.g., "a" and "b"). Each grammar production is of the form A -> $[\ldots]$; where $\mathrm{A}$ is a nonterminal and ... is a sequence of any nonterminal or terminal symbol separated by one or more blanks. We also allow A $\rightarrow$ [ . . , ... $]$; to express two grammar productions with the same left-hand side A. That is, $\mathrm{S} 1->$ [ "a" S1 "a", "b" S1 "b" ]; is syntactic sugar for S1 -> [ "a" S1 "a"]; S1 -> [ "b" S1 "b" ];. Any text after ; ; is considered a comment.

If we type covenant INPUT --gen=max-gen, where max-gen refers to the maximum star-epsilon generalization, we obtain:

$\overline{{ }^{6} \text { COVENANT }}$ has been tested only for x86_64. 


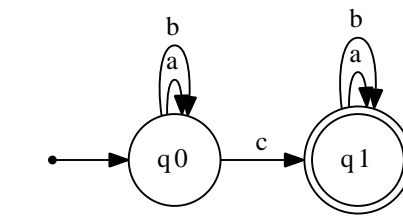

Initial Regular Approximation of L1

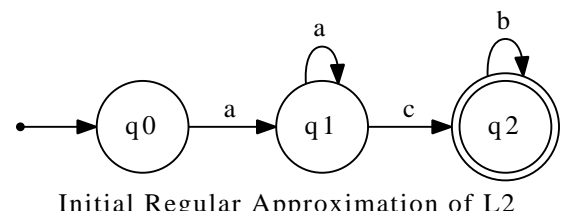

Initial Regular Approximation of L2

Fig. 1: abstractions. dot: initial regular abstractions

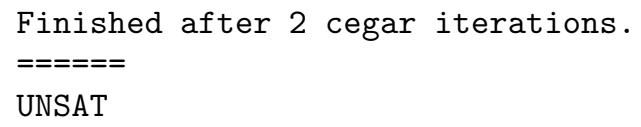

This means that COVENANT proved that the two languages $L_{1}$ and $L_{2}$ are disjoint. Similar result and number of iterations are obtained if we run with option --gen=greedy (for the greedy generalization).

We can also view the dot files produced by COVENANT by adding option --dot. Figure 1 depicts the initial regular approximations of the context-free grammars by means of finite-state automata. Note that in both cases the structure of the original context-free grammar is preserved as much as possible. For instance, for $L_{2}$, the abstraction comes from the need to "forget" the relation between the number of as and bs while still remembering that a symbolic c must separate the as and bs. Each spurious witness found during the refinement loop is also shown as a finite automata as well as their safe generalizations in Figure 2. Note that after the second witness $w \equiv \mathrm{acb}$ is found, COVENANT only needs to compute a safe generalization of $w$ with respect to $L_{1}$, since $w$ is already recognized by $L_{2}$. Figure 3 shows the final regular approximations which prove that $L_{1}$ and $L_{2}$ are disjoint.

"SAT" example. Consider now the languages $L_{1}=\left\{w \mid \# \mathbf{a}\right.$ 's $=\# \mathbf{b}^{\prime} s, w \in$ $\left.\{\mathrm{a}, \mathrm{b}\}^{*}\right\}$ and $L_{2}=\left\{w w^{R} \mid w \in\{\mathrm{a}, \mathrm{b}\}^{*}\right\}$ with the corresponding context-free grammars in COVENANT format:

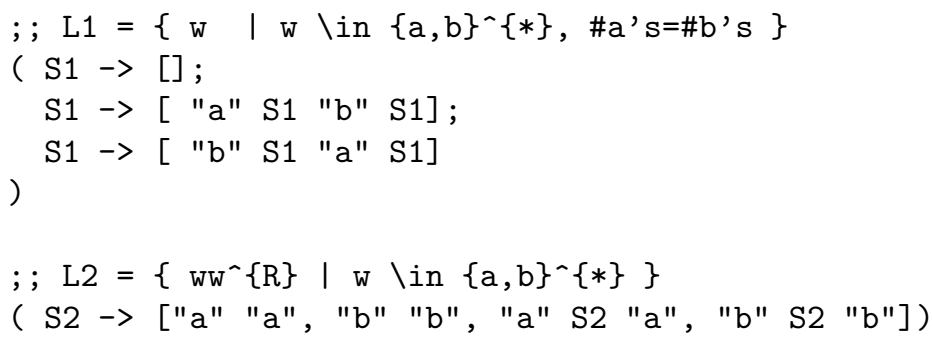




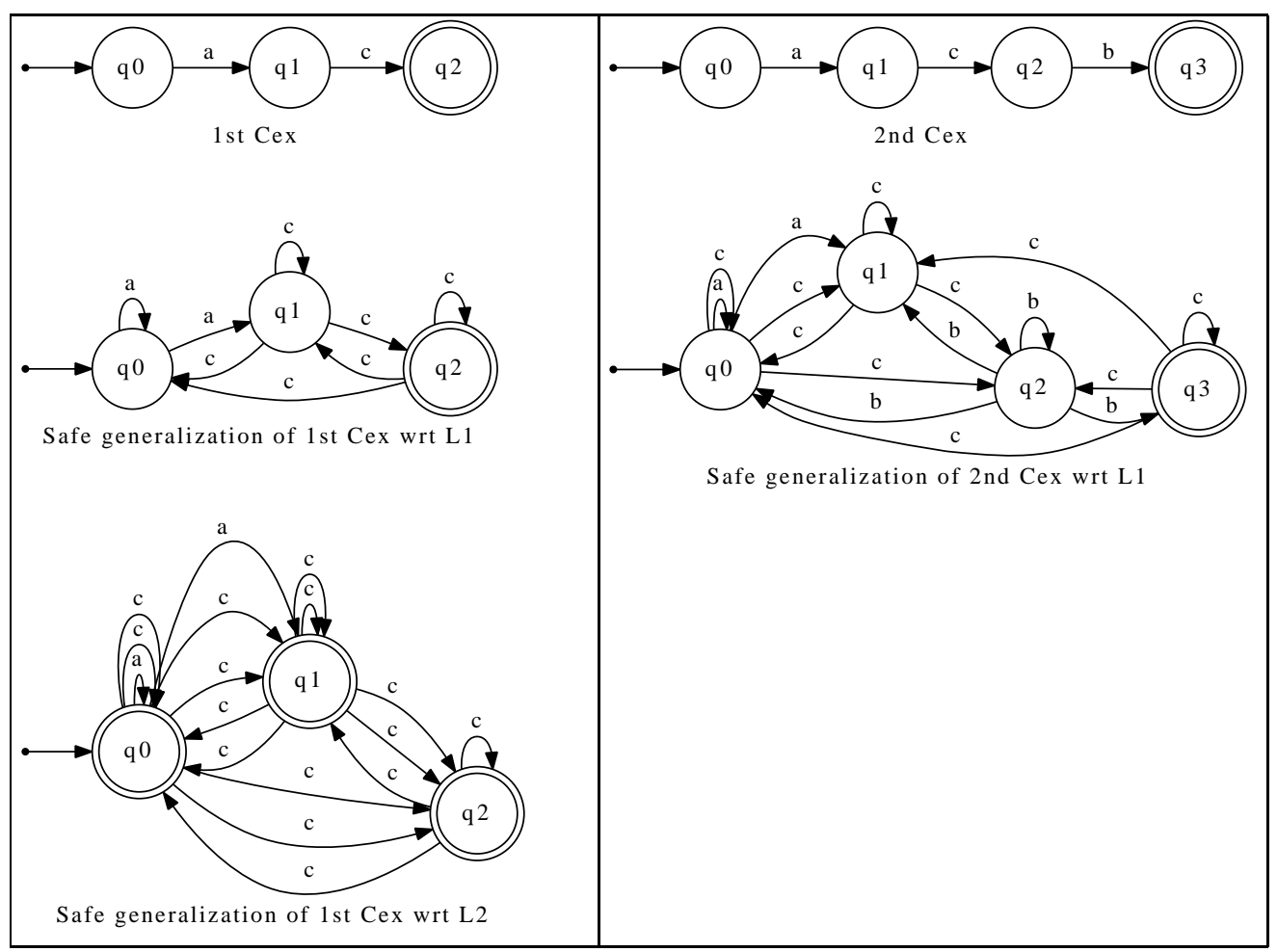

Fig. 2: refinements.dot: witnesses and safe generalizations

By typing covenant INPUT, we obtain:

Found a solution after 5 iterations:

$\mathrm{a} b \mathrm{~b}$ a

$=====$

SAT

$=====$

That is, COVENANT found a word abba that is recognized by both languages. Moreover, we can ask COVENANT for more solutions, say, five more solutions, by typing covenant INPUT --solutions 5:

Found a solution after 5 iterations:

$\mathrm{a} b$ b a

Found a solution after 6 iterations:

b a a b

Found a solution after 20 iterations:

$\mathrm{a} a \mathrm{~b} b \mathrm{~b}$ b a $\mathrm{a}$ 


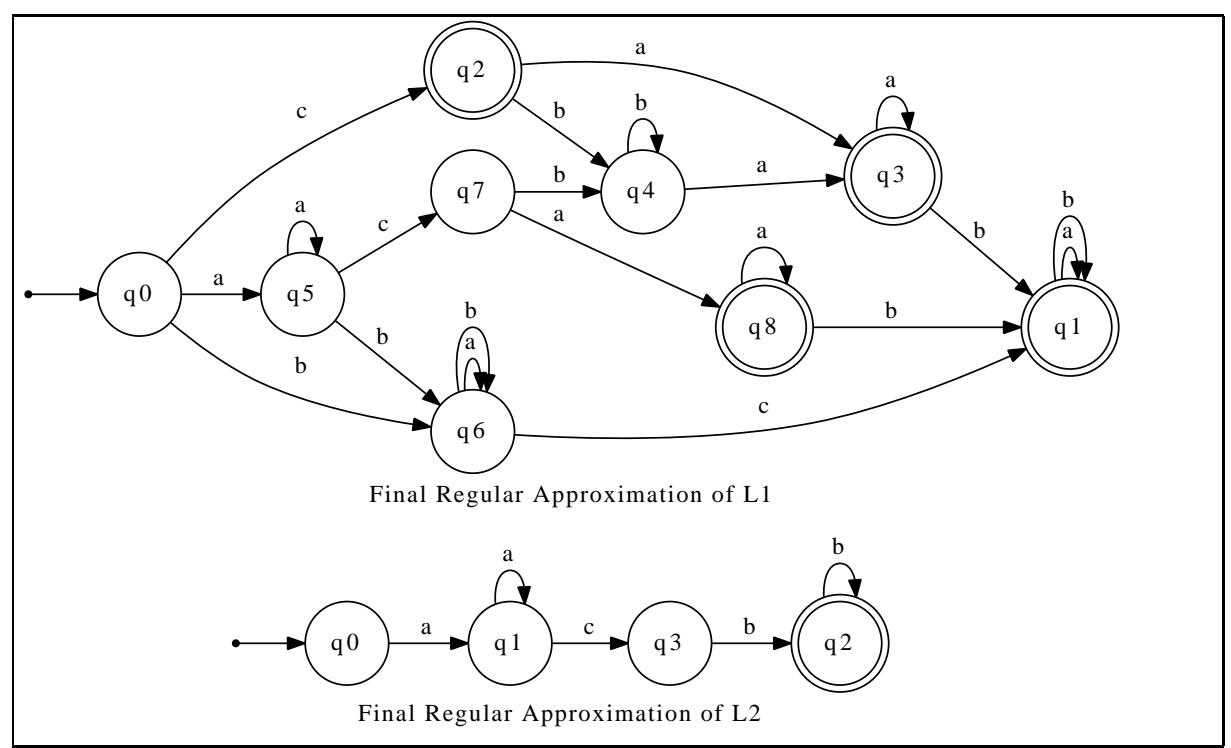

Fig. 3: proofs.dot: final regular abstractions that prove emptiness

Found a solution after 22 iterations:

$\mathrm{a} b$ a $b$ b a b a

Found a solution after 23 iterations:

$\mathrm{a} b \mathrm{~b}$ a $\mathrm{a}$ b b a

$=====$

SAT

$=====$ 